\title{
Cardiovascular research in pathology: where are we now?
}

\section{Editorial}

Cardiovascular disease, whatever approach of study has been conducted - and there are a large number as epidemiological, pathological, clinical, therapeutic, prognostic, and so on - clearly shows morphological and ultrastructural patterns carefully identified, to whom the clinical signs of a specific disorder are met. ${ }^{1}$ In addition, cardiovascular diseases still are one of the most prevalent causes of morbidity and mortality in the western countries. ${ }^{2,3}$

It is worth noting that a medical research recognizes several steps to provide reliable data with regard to a studied topic. In addition, there is evidence that when the result of an opinion poll is announced the sample size of the studied parameter should be given. ${ }^{4}$

The first step to be conducted is analyzing the characteristics of a disease in a single individual and, then, in a large number of subjects affected by the same disorders (sample size). When a common occurrence of observations can be noted in almost all individuals we can conclude the second step (statistical opinion) consisting of the assessment of the type and outcome of the analyzed disorder.

The third step allows to classify the symptoms and basic alterations, which accompany the disease studied (analysis of the results).

Finally, a fourth phase of the research conducted permits to group all diseased subjects showing the same disorders in the context of the specific disease (standardized results).

Following this pattern, it is worthy of mention the degree now reached by the cardiovascular research. The observations related to the ischemic heart disease allow establishing that 3 factors of pathology have been clearly demonstrated by the findings on the subject: atherosclerotic coronary occlusion with or no complications, types of necrosis observed in the acute myocardial infarction as coagulative, colliquative and myocytolysis, and no or minimal morphological alterations of the myocardium when an individual meets sudden cardiac death. ${ }^{5-8}$

Morpho-pathological alterations of hypertension can be minimal or absolutely absent unless a thickening of the arterial wall. On the contrary, when elevated blood pressure associated with microvascular pathology and organ damage, severe lesions, which depend on both degree of hypertension and altered function of body organs affected, are clearly seen with a significant impact to determine the prognosis of the individuals showing these disorders. ${ }^{9-11}$ Heart, brain, and, often, kidney are the pathological targets in the subjects suffering from severe and malignant hypertension. Artery vessel occlusion at great arteries and microcirculation can be identified as the most severe alterations able to cause organ damage in those individuals with complicated hypertension.

With regards to the morpho-pathological alterations of the congenital malformations of the heart and great vessels, valvular disorders, and pericardial disease, there is evidence that well
Volume 8 Issue 5 - 2017

\author{
Aurelio Leone $e^{1,2,3}$ \\ 'Fellow of the American Society of Hypertension (FASH), USA \\ ${ }^{2}$ Fellow of the Royal Society for Promotion of Health (FRSPH), \\ UK \\ ${ }^{3}$ Editor-in-Chief of the Journal of Cardiology \& Current \\ Research, USA
}

Correspondence: Aurelio Leone, Fellow of the American Society of Hypertension (USA), Fellow of the Royal Society for Promotion of Health (UK), Editor-in-Chief of the Journal of Cardiology \& Current Research (USA),Via Provinciale 27, 19030 Castelnuovo Magra, Italy, Email reliol@libero.it

Received: April 17, 2017| Published: April 26, 2017

established pathology has been described in the past ${ }^{12}$ similar to that of the current observation.

A different approach requires the pathology of heart muscle, primarily myocarditis and hypertrophic cardiomyopathy. ${ }^{13,14}$

Myocarditis is an inflammatory disease of the myocardium with a wide range of clinical presentations, from subtle to devastating results that can also cause death as a final result. On the contrary, hypertrophic cardiomyopathy does not show inflammatory patterns, but is an inherited intrinsic disease of the myocardium characterized by left ventricular hypertrophy without chamber dilatation in the absence of either a systemic or other cardiac disease. There is evidence that this field of cardiac pathology still requires further studies to clarify the true significance and extent of these diseases.

\section{Conclusion}

From the described observations, it is worth noting that the knowledge of cardiac pathology follows two clearly defined ways. The first, mainly related to valvular components of the heart, hypertension, and ischemic heart disease, is characterized by histopathologic patterns completely acquired, while continuously newer results update the alterations of myocardial cells when cardiac muscle is primarily involved with non-vascular mechanisms. For example, genetic pathology of the heart is a very encouraging field of investigations, which promise a more significant approach to define cardiac alterations.

\section{Acknowledgments}

None. 


\section{Conflicts of interest}

Author declares there are no conflicts of interest.

\section{Funding}

None.

\section{References}

1. Leone A. Ultrastructural Components of Myocardial Cell: What is their Role in Myocardial Pathology? J Cardiol Curr Res. 2007;8 (3):00283.

2. Leone A. Relationship between cigarette smoking and other coronary risk factors in atherosclerosis: risk of cardiovascular disease and preventive measures. Curr Pharm Des. 2003;9(29):2417-2423.

3. WHO. The global burden of disease: 2004 update. Geneva: World Health Organization. 2004.

4. Porkess R. The Harper Collins Dictionary of Statistics. Harper Perennial, A Division of Harper Collins Publishers. 1991.

5. Libby P, Theroux P. Pathophysiology of coronary artery disease. Circulation. 2005;111:3481-3488.

6. Falk E. Unstable angina with fatal outcome: dynamic coronary thrombosis leading to infarction and/or sudden death: autopsy evidence of recurrent mural thrombosis with peripheral embolization culminating in total vascular occlusion. Circulation. 1985;71(4):699-708.
7. Leone A. Myocardial Infarction. Pathological Relevance and Relationship with Coronary Risk Factors. Curr Pharm Des (in press). 2017.

8. Zipes DP, Wellens HJJ. Sudden cardiac death. Circulation. 1998;98:2334-2351.

9. Weller RO. Vascular pathology in hypertension. Age Ageing. 1979;8(2):99-103.

10. Fujimoto T. Pathology of malignant nephrosclerosis with special reference to the difference between histologic manifestations of pure exacerbated forms. Tohoku J Exp Med . 1978;125(2):135-153.

11. Lawes CM, Vander Hoorn S, Rodgers A. Global burden of bloodpressure-related disease. Lancet. 2001;371(9623):1513-1518.

12. Askey JM, Kahler JE. Longevity in extensive organic heart lesions: a case of Lutembacher's syndrome in a man aged 72. Ann Intern Med. 1950;33(4):1031-1036.

13. Aretz HT, Billingham ME, Edwards WD, et al. Myocarditis: A histopathologic definition and classification. Am J Cardiovasc Path. 1987;1(1):3-14.

14. Hughes SE. The pathology of hypertrophic cardiomyopathy. Histopathology. 2004;44(5):412-427. 